\title{
The fate of silver nanoparticles in authentic human saliva
}

Kamonwad Ngamchuea, Christopher Batchelor-McAuley, Richard G. Compton*

*corresponding author: Richard G. Compton, Department of Chemistry, Physical \& Theoretical Chemistry Laboratory, University of Oxford, South Parks Road, Oxford, OX1 3QZ, United Kingdom

Email: richard.compton@ chem.ox.ac.uk. Tel: +44(0)1865275957 Fax: +44(0)1865275410

\begin{abstract}
The physicochemical properties of silver nanoparticles (AgNPs) in human whole saliva are investigated herein. In authentic saliva samples, AgNPs exhibit a great stability with over $70 \%$ of the nanomaterial remaining intact after a 24-hour incubation in the presence of $\sim 0.3 \mathrm{mM}$ dissolved oxygen. The small loss of AgNPs from the saliva sample has been demonstrated to be a result of two processes: agglomeration/aggregation (not involving oxygen) and oxidative dissolution of AgNPs (assisted by oxygen). In authentic saliva, AgNPs are also shown to be more inert both chemically (silver oxidative dissolution) and electrochemically (electron transfer at an electrode) than in synthetic saliva or aqueous electrolytes. The results thus predict based on the chemical persistence (over a 24-hour study) of AgNPs in saliva and hence the minimal release of hazardous $\mathrm{Ag}^{+}$and reactive oxygen species that the AgNPs are less likely to cause serious harm to the oral cavity but this persistence may enable their transport to other environments.
\end{abstract}

\section{Keywords}

Saliva; silver nanoparticles; oxidation; dissolution; agglomeration 


\section{Main text}

As one of the most commonly used nanoparticles, a considerable quantity of nano-silver has been accumulated in water, soil, plants and animals in the food chain. ${ }^{1-3}$ Silver nanoparticles (AgNPs) are further introduced in the processing, packaging of food containers and the food itself, ${ }^{4,5}$ as well as in drug delivery and biomedical applications. ${ }^{6}$ Direct exposure of the oral cavity to AgNPs is thus unavoidable. Diseases and abnormal activities of internal organs as a result of oral exposure to AgNPs have been reported. ${ }^{7-10}$ The local susceptibility to damage caused by AgNPs in the oral cavity however has not been investigated. In this work, the physicochemical behavior of AgNPs is studied in-vitro in human whole saliva.

The metabolic process that gives rise to the toxicity of AgNPs has yet to be fully elucidated. The potential causes of AgNPs toxicity are suggested to be the interaction with the cell walls, the release of silver(I) ions $\left(\mathrm{Ag}^{+}\right)$and the generation of reactive oxygen species (e.g. $\mathrm{H}_{2} \mathrm{O}_{2}$, $\mathrm{O}_{2}{ }^{-}$, etc.), leading potentially to damage of DNA and important proteins. ${ }^{11-14}$ The toxic pathways mentioned above are strongly dependent on the chemical environments such as $\mathrm{pH}$, concentrations of electrolytes and the presence of molecules that could interact with the silver or reactive oxygen species. ${ }^{15}$

In the oral cavity, saliva is the major component for the maintenance of oral health. It is composed of ca. 99\% water, inorganic and organic substances, enzymes, mucus and glycoproteins. ${ }^{16,17}$ The $\mathrm{pH}$ of saliva is $\sim 6.8$. To understand the toxicity of AgNPs to the oral cavity, one needs to directly investigate the course and fate of AgNPs in saliva. Hitherto only synthetic saliva, to the best of the authors knowledge, has been used to study the properties of the AgNPs. ${ }^{18,19}$ The work presented herein however shows that the chemical and electrochemical behaviors of relevant species in synthetic saliva are significantly different from authentic saliva.

In this study, synthetic saliva (according to DIN 53160-1) was obtained from Synthetic Urine e.K., Germany. Authentic saliva samples were kindly donated by three consenting healthy volunteers without any known pathological conditions and were pooled for the measurements. Further details of authentic saliva collection are provided in the SI.

The physicochemical properties including size, concentration, stability, integrity and electroactivity of AgNPs in human whole saliva are evaluated and discussed on the basis of 
electrochemical and spectrophotometric arguments. The dissolution and hence toxicity of these materials is intimately associated with their redox behaviors. The electrochemical responses of AgNPs oxidation were investigated in authentic saliva samples in comparison with synthetic saliva and standard potassium chloride $(\mathrm{KCl})$ solutions. The oxidation process was studied by two techniques: anodic stripping and nano-impact electrochemistry (single particle coulometry). The solution-phase stability and chemical activity of the AgNPs were investigated by UV-visible spectrophotometric measurements.

In "anodic stripping" studies, an aliquot of AgNPs suspension consisting of $2.4 \times 10^{-16}$ moles of the nanoparticles was dropcast onto the surface of a freshly polished glassy carbon electrode to yield a coverage (projected surface area of AgNPs:electrode) of ca. $4.5 \%$. The modified electrode was allowed to dry under a flowing nitrogen atmosphere. The AgNPmodified electrode was then transferred to authentic saliva, synthetic saliva or standard $\mathrm{KCl}$ solutions $(0,1.0,5.0,10.0$ and $20.0 \mathrm{mM})$, where the electrode was subjected to cyclic voltammetry measurements initially scanning anodically in the potential range of $-0.2 \mathrm{~V}$ to $1.0 \mathrm{~V}$ vs. $\mathrm{Ag} / \mathrm{AgCl}$ (in $3.4 \mathrm{M} \mathrm{KCl}$ ) leakless reference electrode at the scan rate of $10 \mathrm{mV} \mathrm{s}^{-1}$. Figure 1 presents the voltammograms of AgNP anodic stripping in the different samples. A well-defined oxidative response of AgNPs can be obtained in authentic, whole saliva without any pre-treatment or added supporting electrolyte. The anodic stripping peak potentials appear at $\sim 0.18 \mathrm{~V}$ in both synthetic and authentic saliva, which is the same potential as the oxidation of $\mathrm{AgNP}$ in $1.0 \mathrm{mM} \mathrm{KCl}$ solution. At higher chloride concentrations, the positions of the anodic stripping peaks occur at less positive potentials, in agreement with literature..$^{20,21}$

Below, the results as presented as 'mean \pm standard deviation.' In standard $\mathrm{KCl}$ solutions for all the concentrations studied, the total charge $(Q)$ transferred during the oxidation of the immobilized AgNPs was in the range of $16 \pm 5 \mu \mathrm{C}$, corresponding to $22 \pm 6 \%$ of the expected charge $(\sim 75 \mu \mathrm{C}$, see SI for details). In synthetic saliva, the charge transferred was $12 \pm 1 \mu \mathrm{C}$, comparable to that obtained in the $\mathrm{KCl}$ solutions. However, the observed charge transferred in authentic saliva was $2.1 \pm 0.6 \mu \mathrm{C}$ significantly smaller than in the other cases, and hence suggests the more electrochemically inert nature of AgNPs in authentic saliva, vide infra. 


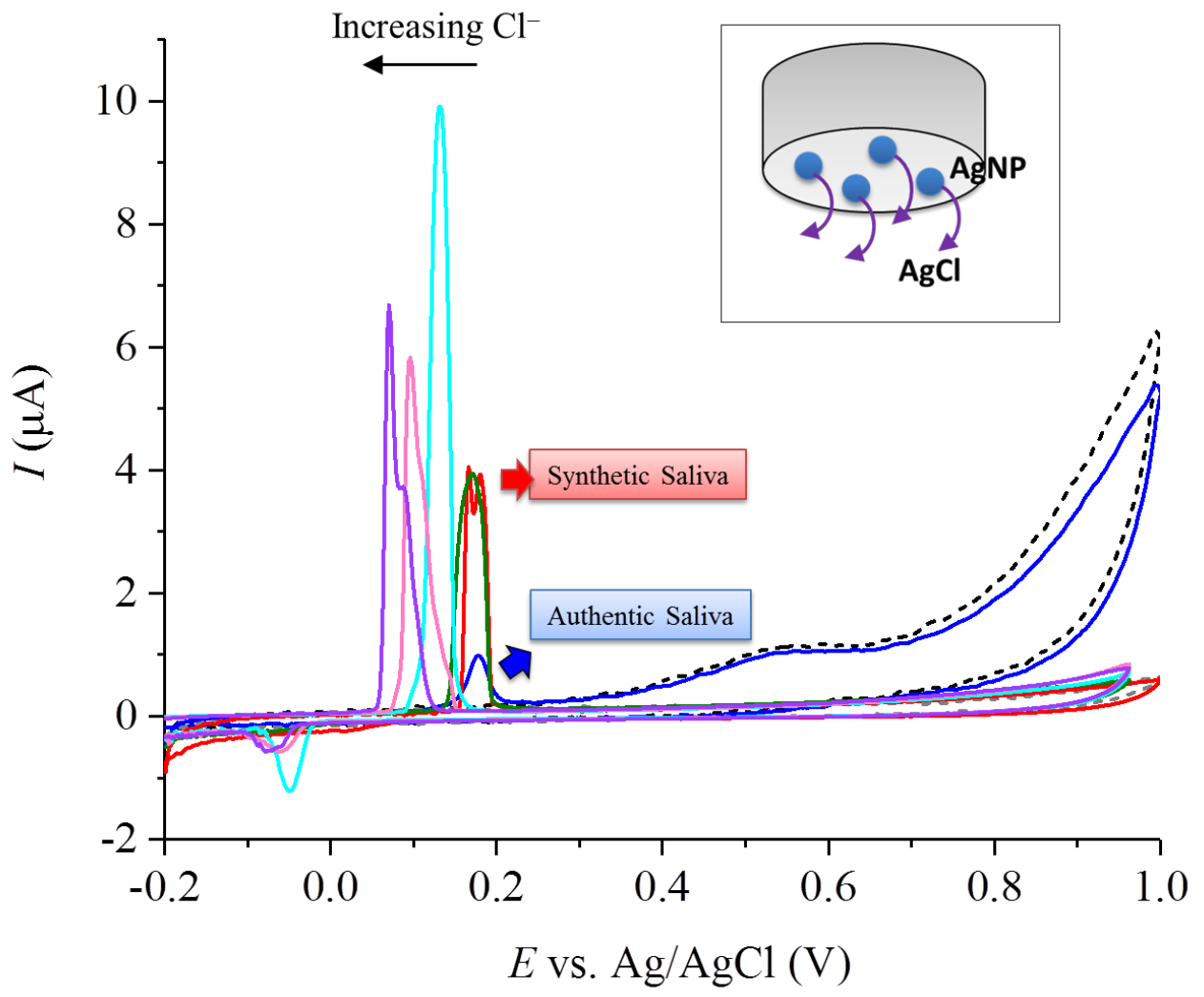

Figure 1: Anodic stripping voltammograms of silver nanoparticles (AgNPs) immobilized on the surface of a glassy carbon electrode in potassium chloride solutions: $1.0 \mathrm{mM}$ (green), $5.0 \mathrm{mM}$ (light blue), $10.0 \mathrm{mM}$ (pink) and $20.0 \mathrm{mM}$ (purple), synthetic saliva (red), and authentic saliva (dark blue). Scan rate of $10 \mathrm{mV} \mathrm{s}^{-1}$. Dashed lines show the voltammograms of synthetic saliva (grey) and authentic saliva (black) at a blank, unmodified electrode. Inlay: Schematic presentation showing AgNPs immobilized on the electrode surface and oxidized (anodic stripping) to form $\mathrm{AgCl}$.

In addition to anodic stripping voltammetry, nano-impact experiments were performed to study the electrochemical responses of AgNPs oxidation in-situ. In solution, AgNPs diffuse freely by virtue of Brownian motion. In the event that a AgNP arrives at the electrode held at a suitable potential $(E>\sim 0.2 \mathrm{~V}$ ), the AgNP is fully oxidized resulting in a current-transient spike. $^{20,22-25}$ The charge transfer for each spike corresponds to the size of the AgNPs, while the diffusion-limited frequency of spikes is indicative of the concentration and agglomeration state of AgNPs in the suspension.

Figure 2 displays examples of cyclic voltammetry responses of a carbon microdisk electrode (33 $\mu \mathrm{m}$ diameter) immersed in a $12 \mathrm{pM}$ AgNP suspension (equivalent to $7.25 \times 10^{-9}$ particles $\mathrm{mL}^{-1}$ ), and the inlays clarify the representative spike shapes. This concentration of AgNPs was chosen for optimal conditions of the nano-impact measurements. In $20.0 \mathrm{mM} \mathrm{KCl}$, the spikes were observed at the frequency of $4.6 \pm 0.6 \mathrm{~s}^{-1}$ (diffusion-limited frequency: $6.2 \mathrm{~s}^{-1}$ ) 
with the average charge transferred per spike of $0.32 \pm 0.23 \mathrm{pC}$. These charges, when corrected by a factor of 0.605 to account for the quasi-spherical nature of the nanoparticles, ${ }^{26}$ correspond to the particle diameters of $48 \pm 9 \mathrm{~nm}$ in good agreement with the sizes obtained from transmission electron microscope (TEM) of $47 \pm 6 \mathrm{~nm}$ (see SI). In synthetic saliva, the features were similar to that in $20.0 \mathrm{mM} \mathrm{KCl}$ with the frequency of spikes of $4.1 \pm 2.2 \mathrm{~s}^{-1}$ and the average charge per spike of $0.31 \pm 0.21 \mathrm{pC}$. In contrast, no spikes were observed in authentic saliva samples. A small number of current-transient spikes with a frequency of $0.13 \pm 0.08 \mathrm{~s}^{-1}$ were observed when authentic saliva was diluted (using synthetic saliva) by a factor of 100. The mean charge transferred per spike in diluted authentic saliva sample is also found to be significantly smaller, $0.19 \pm 0.13 \mathrm{pC}$. The small charges and low response rate of AgNPs oxidation further support the hypothesis that AgNPs are relatively oxidatively inert in authentic saliva. Additional evidences and mechanisms are proposed below.

a)

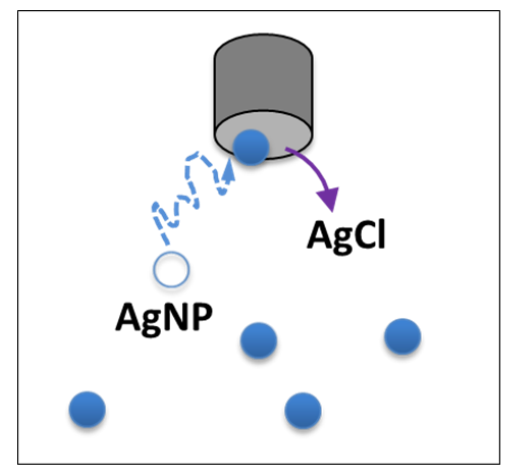

c) Synthetic Saliva

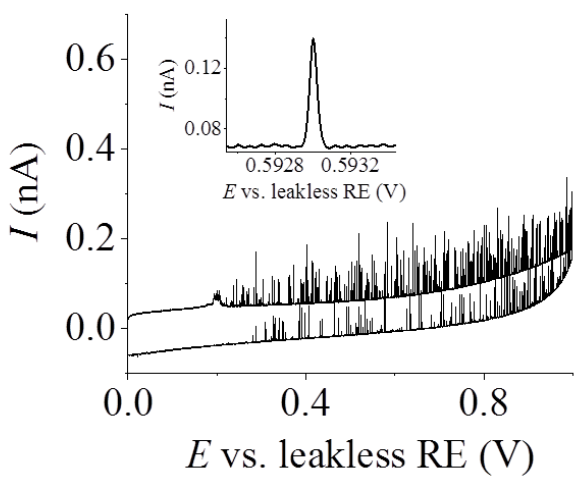

b) $20 \mathrm{mM} \mathrm{KCl}$

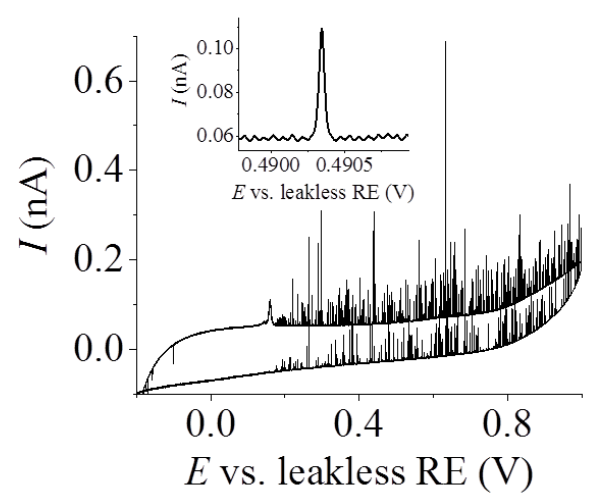

d) Authentic Saliva diluted 1/100 in synthetic saliva

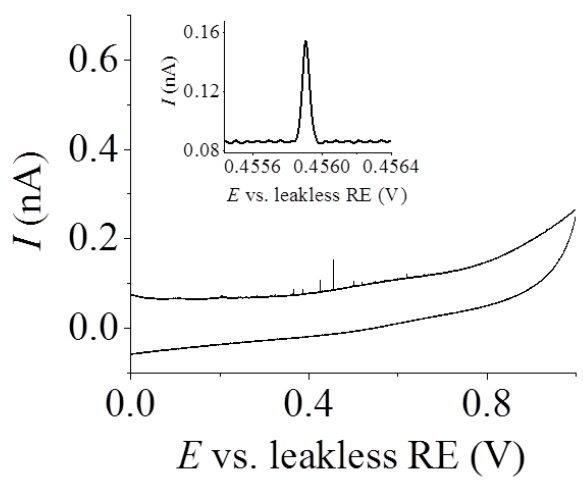

Figure 2: a) Schematic diagram showing AgNPs diffusing freely in solution and oxidizing to $\mathrm{AgCl}$ as the particle hits the electrode. b-d) Cyclic voltammograms showing AgNPs nano-impact spike features in b) $20.0 \mathrm{mM} \mathrm{KCl}$, c) synthetic saliva and d) authentic saliva diluted by a factor of 100 in synthetic saliva. The nano-impact in authentic saliva without dilution shows no spikes (SI). The inlays in figures b-d show representative shapes of the current-transient spikes as a result of single AgNP oxidation. 
The stability of AgNPs in saliva specimens was further investigated by spectrophotometry. AgNPs display plasmonic responses where the position $\left(\lambda_{\max }\right)$ and the magnitude of the peak (maximum absorbance; $\mathrm{A}_{\max }$ ) are related to size and concentration of the particles respectively. ${ }^{27}$ Consequently, the AgNPs (12 $\mathrm{pM}$ or $7.25 \times 10^{-9}$ particles $\mathrm{mL}^{-1}$ ) were incubated in synthetic and authentic saliva at $25{ }^{\circ} \mathrm{C}$, and the spectra recorded as a function of time.

Figure 3 presents the absorption spectra of the AgNPs in synthetic and authentic saliva samples before and after four hours incubation in the absence and presence of atmospheric oxygen ( $0.3 \mathrm{mM}$ dissolved oxygen) ${ }^{28}$ In synthetic saliva in the presence of dissolved oxygen, the maximum absorbance and hence the number of AgNPs in the sample decreased by $81 \%$ in four hours. ${ }^{\dagger 27}$ In authentic saliva, the concentration of AgNPs also decreased in the first four hours in the presence of oxygen, but to a much smaller extent of $27 \%$. This indicates that there are (by a factor of three) more intact AgNPs left in authentic saliva than in synthetic saliva when the AgNPs are incubated in the samples for the same duration. The responses did not decrease further when left for longer (24 hours, SI). The spectrophotometric results thus evidence the greater stability of AgNPs in authentic saliva samples as compared with synthetic saliva.

In the above work, citrate-capped AgNPs were used experimentally. Similar spectrophotometric experiments were performed for AgNPs capped with different molecules including branched polyethylenimine (BPEI), polyethylene glycol (PEG), polyvinylpyrrolidone (PVP) and lipoic acid. In synthetic saliva, the losses of AgNPs after 4-hour incubation under atmospheric oxygen were $61 \%, 85 \%, 4 \%$ and $30 \%$ for BPEI, PEG, PVP and lipoic acid respectively. In authentic saliva, there were 10\%, 11\%, 3\% and 7\% losses of AgNPs capped with BPEI, PEG, PVP and lipoic acid respectively. Consequently, it has been shown that AgNPs were significantly more stable in authentic saliva than in synthetic saliva for all the different capping agents studied (see SI). Note that the AgNPs tested herein are not intended for oral intake, but are the same types as those incorporated in textiles and other commercial products. The findings on the tested AgNPs are likely applicable to the ingested AgNPs. 
$\uparrow$ The number of AgNPs can be interpreted from the value of the maximum absorbance according to BeerLambert law. ${ }^{27}$

The possible origins of the loss of AgNPs UV-vis signal may relate to the agglomeration/ aggregation and/or the dissolution of AgNPs. The latter likely only occurs in the presence of dissolved oxygen (SI). In the absence of oxygen, the loss of AgNPs is attributed to the agglomeration/aggregation of the particles. ${ }^{18,19}$ Consequently, to investigate the effect of oxygen on the disappearance of AgNPs, oxygen was removed from the samples by a strong flow of argon gas into the samples prior to the addition of AgNPs. It was found that after the elimination of oxygen, the AgNPs UV-vis signals still decreased but to much smaller extents of $45 \%$ and $11 \%$ losses of AgNPs after 4 hours incubation in synthetic and authentic saliva respectively (Figure 3). The results thus indicate that both agglomeration/aggregation and oxidative dissolution play important roles in the instability of the AgNPs in solution-phase. The results also indicate that the agglomeration/aggregation of AgNPs progresses to a smaller extent in authentic saliva as compared to synthetic saliva. This conclusion was further corroborated by the observation of the AgNP sizes in authentic vs. synthetic saliva under dark-field microscope (see SI).

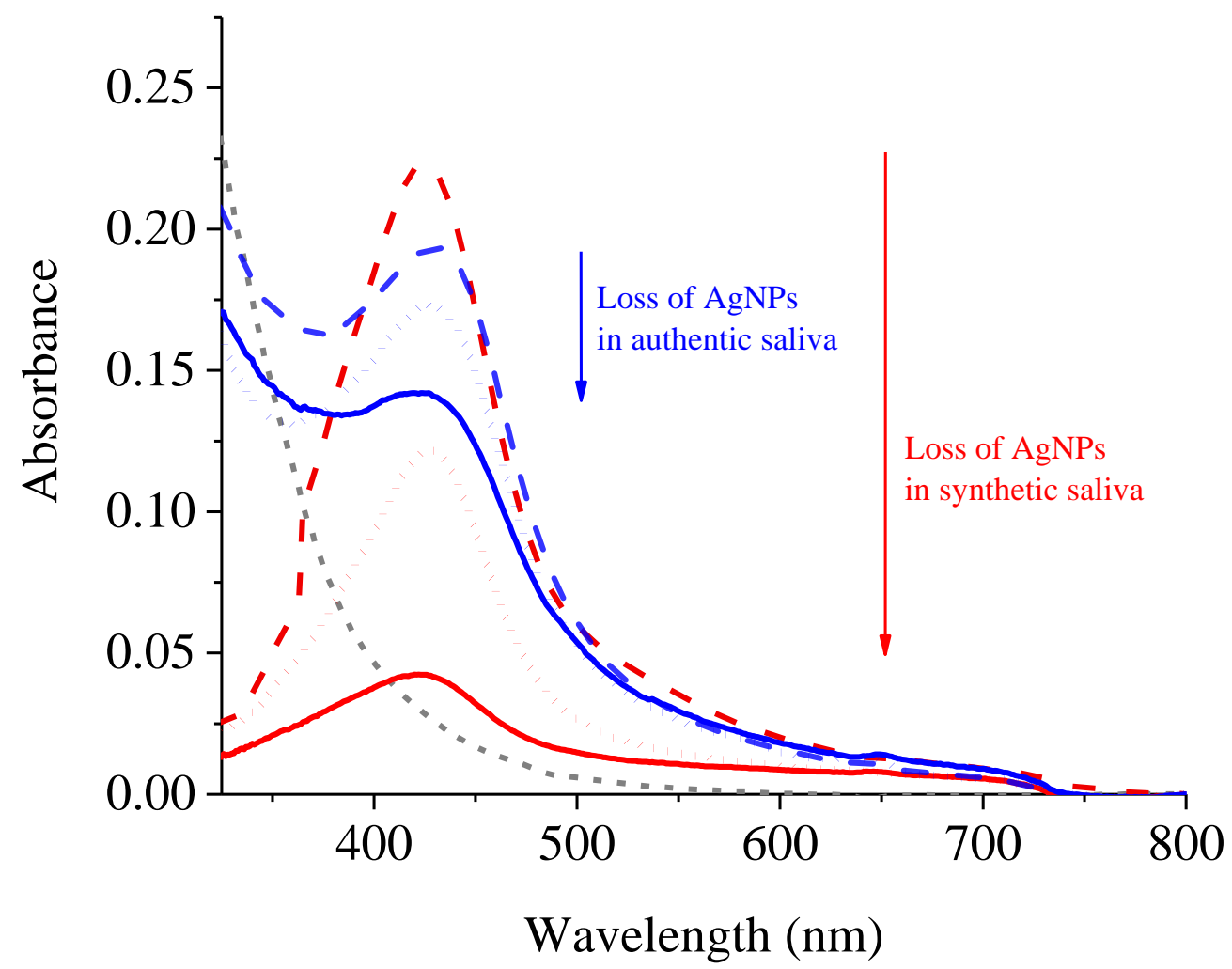


Thus far, the main observations on authentic saliva are that first the voltammetric oxidation of large ensembles of nanoparticles is inhibited in saliva. Second, the frequency of individual nanoparticle oxidations from the solution phase is significantly decreased in the presence of saliva. Moreover even in highly diluted authentic saliva samples, only small oxidative events are observed. Third, the UV-vis studies evidence a low rate of loss of the AgNPs in authentic saliva and that this loss of signal arises from both agglomeration and oxidative dissolution of the nanomaterial. In the presence of oxygen, AgNPs may undergo thermodynamically favorable oxidative dissolution to form $\mathrm{Ag}^{+}$and reactive oxygen species, likely via the following reaction pathways. ${ }^{15}$

$$
\begin{aligned}
& 1 / 2 \mathrm{O}_{2}+\mathrm{H}^{+}+\mathrm{Ag}(\mathrm{s}) \longrightarrow \mathrm{Ag}^{+}+1 / 2 \mathrm{H}_{2} \mathrm{O}_{2} \\
& \mathrm{Ag}(\mathrm{s})+1 / 2 \mathrm{H}_{2} \mathrm{O}_{2}+\mathrm{H}^{+} \longrightarrow \mathrm{Ag}^{+}+\mathrm{H}_{2} \mathrm{O}
\end{aligned}
$$

The toxicity of silver nanoparticles as compared with the bulk silver is thought to result from the efficient divergent diffusion of hydrogen peroxide $\left(\mathrm{H}_{2} \mathrm{O}_{2}\right)$ away from the particle surface, before it can be reduced further to water. ${ }^{14}$

These observations are consistent with the behavior of the nanoparticles in authentic saliva being altered from synthetic saliva or aqueous solutions due to a change in their local chemical environment. This most likely arises from the adsorption of proteins, ${ }^{29}$ large organic fragments or other macromolecules present in authentic saliva onto the surface of the nanoparticles. The surface agents introduce steric stabilization, and hence prevent the formation of clusters. The presence of large organic layer on the AgNPs surface would also be consistent with the redox AgNP dissolution process being inhibited as demonstrated by both the UV-vis and electrochemical studies above, resulting in the greater stability of AgNPs in authentic saliva.

As AgNPs remain intact in authentic saliva samples, it is likely that the particles would pass through the oral cavity into other parts of the body in their original forms. Oral ingestion could therefore be one route by which AgNPs may accumulate in liver, spleen and kidney as observed in laboratory animals. ${ }^{7-10}$ Several in-vivo studies demonstrated DNA damage, permanent genome alterations, changes in blood parameters as well as diseases and abnormal activities of internal organs upon oral intake of AgNPs in mammals. ${ }^{7-10,30}$ These damages are potentially caused partly by reactions with the highly oxidative products of AgNPs dissolution. In-vitro, the toxic effects of AgNPs dissolution on human and animal cells and 
tissues have been validated. ${ }^{11-13}$ It was also suggested that AgNPs dissolution may require cellular internalization. ${ }^{31}$ This could possibly be due to the different modification of the AgNPs surface as in the case of authentic vs. synthetic saliva and/or the different environmental conditions such as $\mathrm{pH}$ (refer to the $\mathrm{H}^{+}$dependence in eqn. 1-2) inside and outside the cells.

In conclusion, this work proves on the basis of the conservation of the nanoparticles integrity that AgNPs are relatively stable in authentic human whole saliva, as compared with synthetic saliva or aqueous solutions. The formation of $\mathrm{Ag}^{+}$and possibly reactive oxygen species in saliva are significantly lower. The presence of AgNPs in the oral cavity, whether by accident or intended for biodiagnostic, therapeutic or drug delivery applications, ${ }^{6}$ is thus to a first approximation predicted not to cause serious harm to the oral cavity. Finally, based on these results any method validations or in-vitro studies related to oral cavity are also recommended to be performed in authentic saliva, as opposed to synthetic saliva because of the significant difference in the physicochemical properties of chemical species in the two media.

\section{Acknowledgement}

KN acknowledges funding from the Royal Thai government (DPST scholarship). This work is supported by the funding from the European Research Council under the European Union's Seventh Framework Programme (FP/2007-2013)/ERC Grant Agreement no. [320403].

\section{Disclosure statement}

The authors declare no conflict of interest.

\section{Supporting Information}

Experimental; AgNPs characterisation; anodic stripping (predicted charges and the effect of prolonged exposure); nano-impacts (calculation of the size of AgNPs and the shell thickness); UV-vis spectra (after 24 hours incubation and AgNPs capped with different molecules); darkfield microscope images of AgNPs in saliva. 


\section{References}

(1) Gottschalk, F.; Sonderer, T.; Scholz, R. W.; Nowack, B. Environ. Sci. Technol. 2009, 43, 9216-9222.

(2) Harris, A. T.; Bali, R. J. Nanopart. Res. 2007, 10, 691-695.

(3) Lankveld, D. P.; Oomen, A. G.; Krystek, P.; Neigh, A.; Troost-de Jong, A.; Noorlander, C.

W.; Van Eijkeren, J. C.; Geertsma, R. E.; De Jong, W. H. Biomaterials 2010, 31, 8350-8361.

(4) Mackevica, A.; Olsson, M. E.; Hansen, S. F. J. Nanopart. Res. 2016, 18.

(5) Carbone, M.; Donia, D. T.; Sabbatella, G.; Antiochia, R. J. King Saud Univ. - Sci. 2016, 28 , 273-279.

(6) Austin, L. A.; Mackey, M. A.; Dreaden, E. C.; El-Sayed, M. A. Arch. Toxicol. 2014, 88, 1391-1417.

(7) Tiwari, R.; Singh, R. D.; Khan, H.; Gangopadhyay, S.; Mittal, S.; Singh, V.; Arjaria, N.; Shankar, J.; Roy, S. K.; Singh, D.; Srivastava, V. Nanotoxicology 2017, 11, 671-686.

(8) Heydrnejad, M. S.; Samani, R. J.; Aghaeivanda, S. Biol. Trace. Elem. Res. 2015, 165, $153-$ 158.

(9) Kim, Y. S.; Song, M. Y.; Park, J. D.; Song, K. S.; Ryu, H. R.; Chung, Y. H.; Chang, H. K.; Lee, J. H.; Oh, K. H.; Kelman, B. J.; Hwang, I. K.; Yu, I. J. Part. Fibre Toxicol. 2010, 7, 20.

(10) Jeong, G. N.; Jo, U. B.; Ryu, H. Y.; Kim, Y. S.; Song, K. S.; Yu, I. J. Arch. Toxicol. 2010, 84, 63-69.

(11) AshaRani, P. V.; Low Kah Mun, G.; Hande, M. P.; Valiyaveettil, S. ACS nano 2009, 3, 279-290.

(12) Carlson, C.; Hussain, S. M.; Schrand, A. M.; Braydich-Stolle, L. K.; Hess, K. L.; Jones, R. L.; Schlager, J. J. J. Phys. Chem. B. 2008, 112, 13608-13619.

(13) Foldbjerg, R.; Dang, D. A.; Autrup, H. Arch. Toxicol. 2011, 85, 743-750.

(14) Batchelor-McAuley, C.; Tschulik, K.; Neumann, C. C. M.; Laborda, E.; Compton, R. G. Int. J. Electrochem. Sci. 2014, 9, 1132-1138.

(15) Liu, J.; Hurt, R. H. Environ. Sci. Technol. 2010, 44, 2169-2175.

(16) de Almeida Pdel, V.; Gregio, A. M.; Machado, M. A.; de Lima, A. A.; Azevedo, L. R. J. Contemp. Dent. Pract. 2008, 9, 72-80.

(17) Ngamchuea, K.; Chaisiwamongkhol, K.; Batchelor-McAuley, C.; Compton, R. G. Analyst 2018, 143, 81-99.

(18) Pindakova, L.; Kasparkova, V.; Kejlova, K.; Dvorakova, M.; Krsek, D.; Jirova, D.;

Kasparova, L. Int. J. Pharm. 2017, 527, 12-20.

(19) Walczak, A. P.; Fokkink, R.; Peters, R.; Tromp, P.; Herrera Rivera, Z. E.; Rietjens, I. M.; Hendriksen, P. J.; Bouwmeester, H. Nanotoxicology 2013, 7, 1198-1210.

(20) Ngamchuea, K.; Clark, R. O. D.; Sokolov, S. V.; Young, N. P.; Batchelor-McAuley, C.; Compton, R. G. Chem. Eur. J 2017, 23, 16085-16096.

(21) Toh, H. S.; Batchelor-McAuley, C.; Tschulik, K.; Compton, R. G. Analyst 2013, 138, 4292-4297.

(22) Bartlett, T. R.; Sokolov, S. V.; Plowman, B. J.; Young, N. P.; Compton, R. G. Nanoscale 2016, 8, 16177-16181.

(23) Toh, H. S.; Jurkschat, K.; Compton, R. G. Chem. Eur. J 2015, 21, 2998-3004.

(24) Saw, E. N.; Kratz, M.; Tschulik, K. Nano Res. 2017.

(25) Brasiliense, V.; Patel, A. N.; Martinez-Marrades, A.; Shi, J.; Chen, Y.; Combellas, C.;

Tessier, G.; Kanoufi, F. J. Am. Chem. Soc. 2016, 138, 3478-3483.

(26) Sokolov, S. V.; Batchelor-McAuley, C.; Tschulik, K.; Fletcher, S.; Compton, R. G. Chem. Eur. J 2015, 21, 10741-10746.

(27) Ngamchuea, K.; Batchelor-McAuley, C.; Sokolov, S. V.; Compton, R. G. Anal. Chem. 2017, 89, 10208-10215. $53-62$.

(28) Truesdale, G. A.; Downing, A. L.; Lowden, G. F. J. Chem. Technol. Biotechnol. 2007, 5,

(29) Duran, N.; Silveira, C. P.; Duran, M.; Martinez, D. S. J. Nanobiotechnol. 2015, 13, 55. 
(30) Kovvuru, P.; Mancilla, P. E.; Shirode, A. B.; Murray, T. M.; Begley, T. J.; Reliene, R. Nanotoxicology 2015, 9, 162-171.

(31) Gonzalez-Carter, D. A.; Leo, B. F.; Ruenraroengsak, P.; Chen, S.; Goode, A. E.;

Theodorou, I. G.; Chung, K. F.; Carzaniga, R.; Shaffer, M. S.; Dexter, D. T.; Ryan, M. P.; Porter, A. E. Sci. Rep. 2017, 7, 42871. 